\title{
A NOTE ON THE $p$-PARABOLICITY OF SUBMANIFOLDS
}

\author{
ANA HURTADO* AND VICENTE PALMER*
}

\begin{abstract}
We give a geometric criterion which shows p-parabolicity of a class of submanifolds in a Riemannian manifold, with controlled second fundamental form, for $p \geq 2$.
\end{abstract}

\section{INTRODUCTION}

A Riemannian manifold $M^{n}$ is called parabolic if it does not admit a nonconstant positive superharmonic function (referred to the 2-Laplacian). Otherwise it is called hyperbolic. In the paper [LS], T. Lyons and D. Sullivan stablished a list of equivalent conditions to check the 2-hyperbolicity, (and hence, the 2- parabolicity) of an oriented Riemannian manifold, under the name of the Kelvin-Nevanlinna-Royden criterion. Following this research, in the paper [GT], V. Gol'dshtein and M. Troyanov extended this criterion to the $p$-Laplace operator, $(1<p<\infty)$.

Among the characterizations given by the Kelvin-Nevanlinna-Royden criterion for $p$-parabolicity of a Riemannian manifold, we shall use in this paper those that states that a manifold $M$ is $p$-parabolic, (with $1<p<\infty$ ), if and only if it has vanishing $p$-capacity, namely, there exists a non-empty precompact open set $D \subseteq M$ such that $\operatorname{Cap}_{p}(D, M)=0$.

Here, the $p$-capacity of $D$ is defined by

$$
\operatorname{Cap}_{p}(D, M)=\inf _{u} \int_{M}\|\nabla u\|^{p} d \mu,
$$

where the infimum is taken over all real-valued functions $u \in C_{0}^{\infty}(M)$, with $u \geq 1$ in $D$, (see $[\mathrm{HKM}])$.

On the other hand, in [GT] and [T1] it is possible to find a set of geometric criteria for this so-called type problem for the $p$-Laplacian in Riemannian

2000 Mathematics Subject Classification. Primary 53C40, 31C12; Secondary 53C21, $31 \mathrm{C} 45,60 \mathrm{~J} 65$.

Key words and phrases. Submanifolds, transience, $p$-Laplacian, hyperbolicity, parabolicity, capacity, Hessian-Index comparison theory.

* Work partially supported by the Caixa Castelló Foundation, DGI grant MTM200762344 and Junta de Andalucía grants P06-FQM-01642 and FQM325.

* Work partially supported by the Caixa Castelló Foundation, and DGI grant MTM2007-62344. 
manifolds, namely, to decide when a Riemannian manifold is $p$-parabolic or p-hyperbolic.

In particular, in the Corollary 5.2 of [T1], (see too Proposition 4 in [GT]), a characterization of $p$-parabolicity for Riemannian manifolds with a warped cilindrical end is presented, and in Corollary 5.4 of [T1], (see too Proposition 3 in $[\mathrm{GT}]$ ), we have a sufficient condition for $p$-parabolcity in terms of the volume growth of the manifold.

While these two criteria are intrinsic, we are going to present in this paper a geometric criterion to decide if a submanifold $S^{m}$ properly immersed in an ambient manifold $N^{n}$ with a pole $o$ is $p$-parabolic, which involves (lower) bounds for the mean curvature and the second fundamental form of $S$.

This criterion is based, (as in [MP1] and, specially, [HMP] from which this paper can be considered a spin-off), on The Hessian-Index analysis of the (restricted to $S$ ) extrinsic distance function from the pole, (see [GreW]), and consists basically in two main results, Theorem 4.1 and Theorem 4.2. Both theorems encompasses the notion of comparison constellation to describe the set of sufficient conditions which guarantees p-parabolicity of the submanifold $S^{m}$.

A comparison constellation is a triple $\left\{N^{n}, S^{m}, M_{w}^{m}\right\}$, where $S^{m}$ is the submanifold, $N^{n}$ the ambient manifold and $M_{w}^{m}$ denotes a model space $M_{w}^{m}$ where $w$ is the radial warping function of the metric defined on it, which determines the lower bound for the sectional curvatures of $N$ stated as hypothesis. Moreover, the submanifold is assumed to have lower bounds on the radial part of the mean curvature vector field and on the radial part of the second fundamental form. All these conditions are common for a comparison constellation with lower tangency and a comparison constellation with upper tangency.

However, while in the case of comparison constellations with upper tangency these are the only inequalities required, to define the comparison constellations with lower tangency it is necessary one condition more, namely, that the norm of the tangent component of the unitary intrinsic gradient of the distance from the pole in the ambient manifold, (a function defined on $S$ that we call tangency), is bounded from below by a positive radial function. In this sense, and as we shall see with more detail in Subsection 3.3, every comparison constellation with lower tangency is a comparison constellation with upper tangency.

Finally, it is worthy to say that in both Theorems it must be satisfied an inequality involving the radial bounds for the extrinsic curvatures and the radial function $w$. This inequality is called the balance condition and in Theorem 4.1 is exactly the opposite than in Theorem 4.2.

We can say, having on account all the above considerations, that Theorem 4.1 represents the exact counterpart, (concerning the tangency and the curvature assumptions), to the $p$-hyperbolicity result in $[\mathrm{HMP}]$, (sharing the 
same balance condition). On the other hand, hypothesis in Theorem 4.2 constitutes a new set of geometric sufficient conditions for $p$-parabolicity, which, in its turn, are also related with the hyperbolicity statement in [HMP], as we explain in Remark 4.4.

1.1. Outline of the paper. We shall present the basic definitions concerning the $p$-Laplacian in Section 2. Section 3 is devoted to the study of the curvature setting where our results hold, together with the Hessian and Laplacian analysis needed. Main results and examples are stated and proved in Sections 4, 5, 6 and 7.

1.2. Acknowledgements. We would like to acknowledge professors Ilkka Holopainen and Steen Markvorsen their useful comments concerning these results. We are indebted to the referee for his/her useful observations.

\section{THE $p$-LAPLACIAN}

Let $M$ be a non-compact Riemannian manifold, with the Riemannian metric $\langle\cdot, \cdot\rangle$ and the Riemannian volume form $d \mu$.

The $p$-Laplacian of a $C^{2}$ function $u$ is defined as

$$
\Delta_{p} u=\operatorname{div}\left(\|\nabla u\|^{p-2} \nabla u\right) .
$$

When $p=2$, we have the usual Laplacian, and the classical potential theory developed from the study of the solutions of the Laplace equation

$$
\Delta_{2} u=0 .
$$

However, when $p \neq 2$, equation

$$
\Delta_{p} u=0,
$$

is nonlinear and degenerates at the zeroes of the gradient of $u$.

Then, the solutions of (2.1) need not be smooth, nor even $C^{2}$ and equation (2.1) must be interpreted in a weak sense.

In this way, and given $1<p<\infty$, we say that a function $u \in W_{\text {loc }}^{1, p}(M)$ is a (weak) solution to the $p$-Laplace equation

$$
-\operatorname{div}\left(\|\nabla u\|^{p-2} \nabla u\right)=0
$$

in $M$ if

$$
\int_{M}\left\langle\|\nabla u\|^{p-2} \nabla u, \nabla \phi\right\rangle d \mu=0
$$

for all $\phi \in C_{0}^{\infty}(M)$.

Here, $\nabla u \in L_{\text {loc }}^{1}(M)$ is the distributional gradient of $u \in L_{\text {loc }}^{1}(M)$. Furthermore, $L^{1}(M)$ denotes the space of measurable functions $f: M \longrightarrow \mathbb{R}$ with finite norm $\|f\|_{1}<\infty$, and $L_{\text {loc }}^{1}(M)$ is its corresponding local space defined through the open sets in $M$ with compact closure. In its turn, the space $W^{1, p}(M), 1 \leq p<\infty$ is the Sobolev space of all functions $u \in L^{p}(M)$ 
whose distributional gradient $\nabla u$ belongs to $L^{p}(M)$, equipped with the norm $\|u\|_{1, p}=\|u\|_{p}+\|\nabla u\|_{p}$. The corresponding local space $W_{\text {loc }}^{1, p}(M)$ is defined in an obvious way.

Continuous solutions of (2.2) are called p-harmonic. Here the continuity assumption makes no restriction since every solution of (2.2) has a continuous representative, (see [Se]). The extension of regularity results of this kind, (see $[\mathrm{E}]$ and $[\mathrm{Li}]$ ), from the Euclidean setting to the Riemannian setting is detailed in [HMP], Remark 9.2.

A function $u \in W_{\text {loc }}^{1, p}(M)$ is called a $p$-supersolution in $M$ if

$$
\int_{M}\left\langle\|\nabla u\|^{p-2} \nabla u, \nabla \phi\right\rangle d \mu \geq 0
$$

for all non-negative $\phi \in C_{0}^{\infty}(M)$. If, moreover, $u$ is lower semicontinuous, then $u$ is $p$-superharmonic, (and we denote $\Delta_{p} u \leq 0$ ).

Similarly, a function $v \in W_{\text {loc }}^{1, p}(M)$ is called a $p$-subsolution in $M$ if $-v$ is a $p$-supersolution. If, moreover, $v$ is upper semicontinuous, then $v$ is $p$-subharmonic, $\left(\Delta_{p} v \geq 0\right)$.

A fundamental feature of solutions of (2.2) is the following well-known maximum (or comparison) principle which will be instrumental for the comparison technique presented below in Sections 6 and 7: If $u \in W^{1, p}(M)$ is a $p$ supersolution, $v \in W^{1, p}(M)$ is a $p$-subsolution, and $\max (v-u, 0) \in W_{0}^{1, p}(M)$, then $u \geq v$ a.e. in $M$. In particular, if $D \subset M$ is a precompact open set, $u \in C(\overline{\bar{D}})$ is a $p$-supersolution, $v \in C(\bar{D})$ is a $p$-subsolution, and $u \geq v$ in $\partial D$, then $u \geq v$ in $D$. We refer to [HKM, 3.18] for a short proof of the comparison principle.

\section{Comparison Constellations}

We assume throughout the paper that $S^{m}$ is a non-compact, properly immersed, and connected Riemannian submanifold of a complete Riemannian manifold $N^{n}$. Furthermore, we assume that $N^{n}$ possesses at least one pole. Recall that a pole is a point $o$ such that the exponential map $\exp _{o}: T_{o} N^{n} \rightarrow$ $N^{n}$ is a diffeomorphism. For every $x \in N^{n} \backslash\{o\}$ we define $r(x)=\operatorname{dist}_{N}(o, x)$, and this distance is realized by the length of a unique geodesic from $o$ to $x$, which is the radial geodesic from $o$. We also denote by $r$ the restriction $\left.r\right|_{S}: S \rightarrow \mathbb{R}_{+} \cup\{0\}$. This restriction is called the extrinsic distance function from $o$ in $S^{m}$. The gradients of $r$ in $N$ and $S$ are denoted by $\nabla^{N} r$ and $\nabla^{S} r$, respectively. Let us remark that $\nabla^{S} r(x)$ is just the tangential component in $S$ of $\nabla^{N} r(x)$, for all $x \in S$. Then we have the following basic relation:

$$
\nabla^{N} r=\nabla^{S} r+\left(\nabla^{N} r\right)^{\perp}
$$

where $\left(\nabla^{N} r\right)^{\perp}(x)$ is perpendicular to $T_{x} S$ for all $x \in S$.

With the extrinsic distance at hand, we define the following domains: 
Definition 3.1. Given a connected and complete $m$-dimensional submanifold $S^{m}$ in a complete Riemannian manifold $N^{n}$ with a pole $o$, we denote the extrinsic metric balls of (sufficiently large) radius $R$ and center $o$ by $D_{R}(o)$. They are defined as any connected component of the intersection

$$
B_{R}(o) \cap S=\{x \in S: r(x)<R\},
$$

where $B_{R}(o)$ denotes the open geodesic ball of radius $R$ centered at the pole $o$ in $N^{n}$. Using these extrinsic balls we define the $o$-centered extrinsic annuli

$$
A_{\rho, R}(o)=D_{R}(o) \backslash \bar{D}_{\rho}(o)
$$

in $S^{m}$ for $\rho<R$, where $D_{R}(o)$ is the component of $B_{R}(o) \cap S$ containing $D_{\rho}(o)$.

Remark 3.2. We must point out that these extrinsic domains are precompact, (because the submanifold $S$ is properly immersed), and that the radii $R$ that produce smooth boundaries $\partial D_{R}(o)$ are dense in $\mathbb{R}$ by Sard's theorem and the Regular Level Set Theorem, because the distance function $r$ is smooth in $N^{n} \backslash\{o\}$, and hence, its restriction to $S,\left.r\right|_{S}$.

\subsection{Curvature restrictions.}

Definition 3.3. Let $o$ be a point in a Riemannian manifold $M$ and let $x \in M \backslash\{o\}$. The sectional curvature $K_{M}\left(\sigma_{x}\right)$ of the two-plane $\sigma_{x} \in T_{x} M$ is then called an o-radial sectional curvature of $M$ at $x$ if $\sigma_{x}$ contains the tangent vector to a minimal geodesic from $o$ to $x$. We denote these curvatures by $K_{o, M}\left(\sigma_{x}\right)$.

Definition 3.4. The o-radial mean convexity $\mathcal{C}(x)$ of $S$ in $N$, is defined as follows:

$$
\mathcal{C}(x)=-\left\langle\nabla^{N} r(x), H_{S}(x)\right\rangle, \quad x \in S,
$$

where $H_{S}(x)$ denotes the mean curvature vector of $S$ in $N$.

Moreover:

Definition 3.5. The o-radial component $\mathcal{B}(x)$ of the second fundamental form of $S$ in $N$, is defined as:

$$
\mathcal{B}(x)=-\left\langle\nabla^{N} r(x), \alpha_{x}\left(U_{r}, U_{r}\right)\right\rangle,
$$

where

$$
U_{r}=\nabla^{S}(r(x)) /\left\|\nabla^{S} r(x)\right\| \in T_{x} S \subset T_{x} N
$$

is the unit tangent vector to $S$ in the direction of $\nabla^{S} r(x)$ (resp. tacitly assumed to be 0 in case $\left.\nabla^{S} r(x)=0\right)$.

Finally,

Definition 3.6. The o-radial tangency $\mathcal{T}(x)$ of $S$ in $N$ is defined as follows:

$$
\mathcal{T}(x)=\left\|\nabla^{S} r(x)\right\|
$$

for all $x \in S$. 
Remark 3.7. When the submanifold $S$ is totally geodesic, then $\nabla^{N} r=\nabla^{S} r$ in all points, and, hence, the tangency $\mathcal{T}(x)=\left\|\nabla^{S} r(x)\right\|=1 \forall x \in S$. On the other hand, and given the starting point $o \in S$, from which we are measuring the distance $r$, we know that $\nabla^{N} r(o)=\nabla^{S} r(o)$, so $\left\|\nabla^{S} r(o)\right\|=$ 1. Therefore, the tangency is always bounded from above by 1 and the difference $1-\left\|\nabla^{S} r\right\|$ quantifies the radial detour of the submanifold with respect the ambient manifold as seen from the pole $o$.

We can control locally this detour impossing a lower bound for this quantity.

Upper and lower bounds of $\mathcal{C}(x), \mathcal{B}(x)$ and $\mathcal{T}(x)$ together with a suitable control on the $o$-radial sectional curvatures of the ambient space will eventually control the $p$-Laplacian of restricted radial functions on $S$. All these bounds determines the notion of comparison constellation which we shall define below.

\subsection{Model spaces.}

Definition 3.8 (See [Gri], [GreW]). A $w$-model $M_{w}^{m}$ is a smooth warped product with base $B^{1}=\left[0, \Lambda[\subset \mathbb{R}\right.$ (where $0<\Lambda \leq \infty)$, fiber $F^{m-1}=\mathbb{S}_{1}^{m-1}$ (i.e. the unit $(m-1)$-sphere with standard metric), and warping function $w:\left[0, \Lambda\left[\rightarrow \mathbb{R}_{+} \cup\{0\}\right.\right.$, with $w(0)=0, w^{\prime}(0)=1$, and $w(r)>0$ for all $r>0$. The point $o_{w}=\pi^{-1}(0)$, where $\pi$ denotes the projection onto $B^{1}$, is called the center point of the model space. If $\Lambda=\infty$, then $o_{w}$ is a pole of $M_{w}^{m}$.

Remark 3.9. The simply connected space forms $\mathbb{K}^{m}(b)$ of constant curvature $b$ are $w$-models as we mentioned in [HMP], (see [GreW] and [Gri]).

Proposition 3.10 (See [GreW] and [Gri]). Let $M_{w}^{m}$ be a w-model with warping function $w(r)$ and center $o_{w}$. The distance sphere of radius $r$ and center $o_{w}$ in $M_{w}^{m}$ is the fiber $\pi^{-1}(r)$. This distance sphere has the constant mean curvature $\eta_{w}(r)=\frac{w^{\prime}(r)}{w(r)}$. On the other hand, the $o_{w}$-radial sectional curvatures of $M_{w}^{m}$ at every $x \in \pi^{-1}(r)$ (for $r>0$ ) are all identical and determined by

$$
K_{o_{w}, M_{w}}\left(\sigma_{x}\right)=-\frac{w^{\prime \prime}(r)}{w(r)} .
$$

3.3. Comparison constellations. We now collect the previous ingredients and formulate the general framework for our $p$-parabolicity comparison result, which results a dual setting with respect to the curvature assumptions stated in $[\mathrm{HMP}]$ to obtain p-hyperbolicity.

Definition 3.11. Let $N^{n}$ denote a Riemannian manifold with a pole $o$. Let $S^{m}$ denote a connected complete submanifold properly immersed in $N^{n}$. Let $M_{w}^{m}$ denote a $w$-model with center $o_{w}$; see Definition 3.8. We shall assume that the $o$-radial sectional curvatures of $N$ are bounded from below by the 
$o_{w}$-radial sectional curvatures of $M_{w}^{m}$ :

$$
K_{o, N}\left(\sigma_{x}\right) \geq-\frac{w^{\prime \prime}(r)}{w(r)}
$$

for all $x$ with $r=r(x) \in[0, R]$.

Then the triple $\left\{N^{n}, S^{m}, M_{w}^{m}\right\}$ is called a comparison constellation with lower tangency on the interval $[0, R]$ if the radial tangency $\mathcal{T}$ and the radial convexity functions $\mathcal{B}$ and $\mathcal{C}$ of the submanifold $S^{m}$ are all bounded from below by smooth radial functions $g(r), \lambda(r)$, and $h(r)$, respectively:

$$
\begin{aligned}
& \mathcal{T}(x) \geq g(r(x)), \\
& \mathcal{B}(x) \geq \lambda(r(x)), \text { and } \\
& \mathcal{C}(x) \geq h(r(x)) \text { for all } x \in S^{m} \text { with } r(x) \in[0, R] .
\end{aligned}
$$

Definition 3.12. We assume the same general hypothesis on $S^{m}$ and $N^{n}$ than in the definition above. The triple $\left\{N^{n}, S^{m}, M_{w}^{m}\right\}$ is called a comparison constellation with upper tangency on the interval $[0, R]$ when the radial convexity functions $\mathcal{B}$ and $\mathcal{C}$ of the submanifold $S^{m}$ are all bounded from below by smooth radial functions $\lambda(r)$, and $h(r)$, respectively:

$$
\begin{aligned}
& \mathcal{B}(x) \geq \lambda(r(x)), \text { and } \\
& \mathcal{C}(x) \geq h(r(x)) \text { for all } x \in S^{m} \text { with } r(x) \in[0, R] .
\end{aligned}
$$

Remark 3.13. As the tangency is, in a natural way, bounded from above by 1 in both kind of comparison constellations, we have that the comparison constellations with lower tangency are comparison constellations with upper tangency too.

3.4. Hessian and Laplacian comparison analysis. The 2 nd order analysis of the restricted distance function $r_{\left.\right|_{P}}$ defined on manifolds with a pole is firstly and foremost governed by the Hessian comparison Theorem A in [GreW]:

Theorem 3.14 (See [GreW], Theorem A). Let $N=N^{n}$ be a manifold with a pole o, let $M=M_{w}^{m}$ denote a $w$-model with center $o_{w}$, and $m \leq n$. Suppose that every o-radial sectional curvature at $x \in N \backslash\{o\}$ is bounded from below by the $o_{w}$-radial sectional curvatures in $M_{w}^{m}$ as follows:

$$
K_{o, N}\left(\sigma_{x}\right) \geq-\frac{w^{\prime \prime}(r)}{w(r)}
$$

for every radial two-plane $\sigma_{x} \in T_{x} N$ at distance $r=r(x)=\operatorname{dist}_{N}(o, x)$ from $o$ in $N$. Then the Hessian of the distance function in $N$ satisfies

$$
\begin{aligned}
\operatorname{Hess}^{N}(r(x))(X, X) & \leq \operatorname{Hess}^{M}(r(y))(Y, Y) \\
& =\eta_{w}(r)\left(1-\left\langle\nabla^{M} r(y), Y\right\rangle_{M}^{2}\right) \\
& =\eta_{w}(r)\left(1-\left\langle\nabla^{N} r(x), X\right\rangle_{N}^{2}\right)
\end{aligned}
$$


for every unit vector $X$ in $T_{x} N$ and for every unit vector $Y$ in $T_{y} M$ with $r(y)=r(x)=r$ and $\left\langle\nabla^{M} r(y), Y\right\rangle_{M}=\left\langle\nabla^{N} r(x), X\right\rangle_{N}$.

Now, let us consider a submanifold $S^{m}$ immersed in a Riemannian ambient manifold $N^{n}$ with pole $o$, and with distance function to the pole $r$. We are going to see how the Hessians (in $S$ and in $N$ ), of a radial function defined in the submanifold are related via the second fundamental form $\alpha$ of the submanifold $S$ in $N$.

Proposition 3.15. Let $N^{n}$ be a manifold with a pole o, and let $S^{m}$ denote an immersed submanifold in $N$. If $\left.r\right|_{S}$ is the extrinsic distance function, then, given $q \in S$ and $X \in T_{q} S$,

$$
\left.\operatorname{Hess}^{S} r\right|_{q}(X, X)=\left.\operatorname{Hess}^{N} r\right|_{q}(X, X)+\left\langle\alpha_{q}(X, X), \operatorname{grad}^{N} r(q)\right\rangle
$$

where $\alpha_{q}$ is the second fundamental form of $S$ in $N$ at the point $q \in S$.

As a consequence,

Proposition 3.16. Let $N=N^{n}$ be a manifold with a pole o, and let $S^{m}$ denote an immersed submanifold in $N$. Let $\left.r\right|_{P}$ be the extrinsic distance function. Let $f: \mathbb{R} \longrightarrow \mathbb{R}$ be a smooth function. Then, given $q \in S$ and $X \in T_{q} S$,

$$
\begin{aligned}
\left.\operatorname{Hess}^{S} f \circ r\right|_{q}(X, X) & =f^{\prime \prime}(r)\left\langle\operatorname{grad}^{N} r, X\right\rangle^{2} \\
& +f^{\prime}(r)\left\{\left.\operatorname{Hess}^{N} r\right|_{q}(X, X)\right. \\
& \left.+\left\langle\left.\operatorname{grad}^{N} r\right|_{q}, \alpha_{q}(X, X)\right\rangle\right\}
\end{aligned}
$$

Now, we can combine Proposition 3.16 with Theorem 3.14 and trace the resulting Hessian comparison statement in an orthonormal basis of $T_{q} S^{m}$, to obtain the following Laplacian inequality:

Proposition 3.17. Let $N^{n}$ be a manifold with a pole o, let $M_{w}^{m}$ denote a $w$-model with center $o_{w}$. Suppose that every o-radial sectional curvature at $x \in N \backslash\{o\}$ is bounded from below by the $o_{w}$-radial sectional curvatures in $M_{w}^{m}$ as follows:

$$
\mathcal{K}(\sigma(x))=K_{o, N}\left(\sigma_{x}\right) \geq-\frac{w^{\prime \prime}(r)}{w(r)}
$$

for every radial two-plane $\sigma_{x} \in T_{x} N$ at distance $r=r(x)=\operatorname{dist}_{N}(o, x)$ from o in $N$. Then we have for every smooth function $f(r)$ with $f^{\prime}(r) \leq$ 0 for all $r$, (respectively $f^{\prime}(r) \geq 0$ for all $r$ ):

$$
\begin{aligned}
\Delta^{S}(f \circ r) \geq(\leq) & \left(f^{\prime \prime}(r)-f^{\prime}(r) \eta_{w}(r)\right)\left\|\nabla^{S} r\right\|^{2} \\
& +m f^{\prime}(r)\left(\eta_{w}(r)+\left\langle\nabla^{N} r, H_{S}\right\rangle\right),
\end{aligned}
$$

where $H_{S}$ denotes the mean curvature vector of $S$ in $N$. 


\section{MAin RESUlts}

Applying the notion of a comparison constellation as defined in the previous section, we now formulate our main $p$-parabolicity results. The proofs are developed through the following sections.

Theorem 4.1. Consider a comparison constellation with lower tangency $\left\{N^{n}, S^{m}, M_{w}^{m}\right\}$ on the interval $[0, \infty[$. Assume further that the functions $h(r)$ and $\lambda(r)$ are balanced with respect to the warping function $w(r)$ by the following inequality:

$$
\mathcal{M}_{p}(r):=(m+p-2) \eta_{w}(r)-m h(r)-(p-2) \lambda(r) \geq 0 .
$$

Let $\Lambda_{g, p}(r)$ denote the function

$$
\Lambda_{g, p}(r)=w(r) \exp \left(-\int_{\rho}^{r} \frac{\mathcal{M}_{p}(t)}{(p-1) g^{2}(t)} d t\right) .
$$

Suppose finally that $p \geq 2$ and that

$$
\int_{\rho}^{\infty} \Lambda_{g, p}(t) d t=\infty
$$

Then $S^{m}$ is p-parabolic.

Theorem 4.2. Consider a comparison constellation with upper tangency $\left\{N^{n}, S^{m}, M_{w}^{m}\right\}$ on the interval $[0, \infty[$. Assume further that the functions $h(r)$ and $\lambda(r)$ are balanced with respect to the warping function $w(r)$ by the following inequality:

$$
\mathcal{M}_{p}(r):=(m+p-2) \eta_{w}(r)-m h(r)-(p-2) \lambda(r) \leq 0 .
$$

Let $\Lambda_{p}(r)$ denote the function

$$
\Lambda_{p}(r)=w(r) \exp \left(-\int_{\rho}^{r} \frac{\mathcal{M}_{p}(t)}{(p-1)} d t\right) .
$$

Suppose finally that $p \geq 2$ and that

$$
\int_{\rho}^{\infty} \Lambda_{p}(t) d t=\infty
$$

Then $S^{m}$ is p-parabolic.

Remark 4.3. It is easy to check that, when $p=2$, the lower bound $\lambda(r(x))$ for the $o$-radial component of the second fundamental form $\mathcal{B}(x)$ is obsolete when we consider a comparison constellation, (with upper or lower tangency), $\left\{N^{n}, S^{m}, M_{w}^{m}\right\}$ on the interval [0, $\infty[$. Moreover, the function $\mathcal{M}_{2}(r)$ becomes in this case

$$
\mathcal{M}_{2}(r)=m\left(\eta_{w}(r)-h(r)\right) .
$$

With this consideration at hand, we can find a version of Theorem 4.1 for $p=$ 2 in the paper [MP1], where it is used a more restrictive balance condition 
which implies condition (4.1). On the other hand, we have, based on the same consideration, a version of Theorem 4.2 for $p=2$ in the paper [EP], where we can find a direct proof and some consequences in connection with [MP1].

Remark 4.4. The bounds on the curvatures in Theorem 4.2 represents too a dual scenario with respect the bounds on the curvatures in Theorem 4.1 in [HMP], when we consider $g(r)=1$ in the statement of this last Theorem. We must remark that, in this case, the balance condition is exactly the opposite in both results.

Corollary 4.5. Consider a comparison constellation with upper tangency $\left\{N^{n}, S^{m}, M_{w}^{m}\right\}$ on the interval $\left[0, \infty\left[\right.\right.$. Assume that $\mathcal{M}_{p}(r) \leq 0$ for all $r>0$ and that the warping function $w(r)$ is bounded from below by a positive constant on $\left[r_{0}, \infty\left[\right.\right.$, for some $r_{0}>0$. Then, $S^{m}$ is p-parabolic.

Proof. To obtain the result it suffices to apply Theorem 4.2, taking into account that, under the hypothesis, $\Lambda_{p}(r) \geq w(r)$ for all $r>0$.

Corollary 4.6. Consider a comparison constellation with upper tangency $\left\{N^{n}, S^{m}, M_{w}^{m}\right\}$ on the interval $[0, \infty[$. Assume further that, given $q \geq 2$, $\mathcal{M}_{q}(r) \leq 0$ for all $r>0$ and $h(r) \leq \eta_{w}(r) \leq \lambda(r)$ for all $r>0$.

Assume furthermore that

$$
\int_{\rho}^{\infty} \Lambda_{q}(t) d t=\infty
$$

Then $S^{m}$ is $p$-parabolic, for all $p \geq q$.

Proof. It is straightforward to see that, if $p \geq q$,

$$
\mathcal{M}_{p}(r)=\mathcal{M}_{q}(r)+(p-q)\left(\eta_{w}(r)-\lambda(r)\right) \leq \mathcal{M}_{q}(r) \leq 0,
$$

since $\eta_{w}(r)-h(r)<0$ for all $r>0$. Then, it is easy to check that, under the hypothesis,

$$
\begin{aligned}
& \frac{\mathcal{M}_{p}(r)}{p-1} \leq \frac{\mathcal{M}_{q}(r)}{q-1}, \\
& \text { so } \int_{\rho}^{\infty} \Lambda_{p}(t) d t \geq \int_{\rho}^{\infty} \Lambda_{q}(t) d t=\infty
\end{aligned}
$$

Applying Theorem 4.2, the result follows.

\section{Examples: P-PARABOlic SURFACES OF REVOlution IN $\mathbb{R}^{3}$}

We consider smooth surfaces of revolution in $\mathbb{R}^{3}$ constructed as follows. In the $(x, z)$-plane we consider the profile generating curve consisting of a smooth curve $\Gamma(u)=(x(u), z(u))$, with $x(u)>0$ for all $u$. Then the parametrization of this revolution surface is given by

$$
\sigma(u, v)=(x(u) \cos v, x(u) \sin v, z(u)) ; \quad v \in[-\pi, \pi] .
$$


In this Section, we are going to check the $p$-parabolicity of three of these surfaces, (the catenoid, the hyperboloid of one sheet and the paraboloid), using Theorem 4.1. We must remark that these surfaces are warped products of the form $\Gamma \times_{x} S_{1}^{1}$, where $\Gamma$ is the generating curve, and hence it is possible to apply Corollary 5.2 in [T1] in order to know if they are $p$-parabolic or not.

Example 5.1. The Catenoid $S$ is a minimal surface and hence $\mathcal{C}(x) \geq 0$. We have as generating curve in this particular case:

$$
\begin{aligned}
& x(u)=\cosh (u) \\
& z(u)=u
\end{aligned}
$$

for $u \in \mathbb{R}$. The parametrization of the surface is:

$$
\sigma(u, v)=(\cosh (u) \cos v, \cosh (u) \sin v, u) ; u \in \mathbb{R} ; \quad v \in[-\pi, \pi] .
$$

Hence the distance function to the origin $p=(0,0,0)$ is given by

$$
r(u, v)=\sqrt{\cosh (u)^{2}+u^{2}} .
$$

On the other hand, it is easy to see that the unit normal vector to the surface, $N(u, v)$, turns out to be

$$
N(u, v)=\frac{1}{\cosh (u)}(-\cos (v),-\sin (v), \sinh (u)),
$$

and then, using that $\nabla^{\mathbb{R}^{3}} r=\frac{\sigma(u, v)}{r(\sigma(u, v))}$,

$$
\begin{aligned}
\nabla^{S} r & =\nabla^{\mathbb{R}^{3}} r-\left\langle\nabla^{\mathbb{R}^{3}} r, N\right\rangle N \\
& =\frac{\sinh (u) \cosh (u)+u}{\cosh ^{2}(u) \sqrt{\cosh ^{2}(u)+u^{2}}}(\sinh (u) \cos (v), \sinh (u) \sin (v), 1)
\end{aligned}
$$

As a consequence, the tangency function is given by

$$
\mathcal{T}(u, v)=\frac{|\sinh (u) \cosh (u)+u|}{\cosh (u) \sqrt{\cosh (u)^{2}+u^{2}}} .
$$

Now, if we compute the second fundamental form of $S$ in $U_{r}(u, v)=$ $\nabla^{S} r /\left\|\nabla^{S} r\right\|$, we have that

$$
\alpha\left(U_{r}, U_{r}\right)=\left\langle\nabla_{U_{r}}^{\mathbb{R}^{3}} U_{r}, N\right\rangle N=-\left\langle U_{r}, \nabla_{U_{r}}^{\mathbb{R}^{3}} N\right\rangle N=-\frac{1}{\cosh ^{2}(u)} N,
$$

and then

$$
B(u, v)=-\left\langle\nabla^{\mathbb{R}^{3}} r, \alpha\left(U_{r}, U_{r}\right)\right\rangle=\frac{u \sinh (u)-\cosh (u)}{\cosh ^{3}(u) \sqrt{u^{2}+\cosh ^{2} u}} .
$$


Before computing the geometric elements needed, it is a simple calculation to see that

$$
\mathcal{T}(u, v) \geq \sqrt{\frac{2 \ln (r(u, v))}{1+2 \ln (r(u, v))}}, \quad B(u, v) \geq \frac{-1}{r(u, v)} \quad \text { for all } u, v .
$$

Therefore, taking $\lambda(r)=-\frac{1}{r}, h(r)=0$ and $g(r)=\sqrt{\frac{2 \ln (r)}{1+2 \ln (r)}}$ we have that the triple $\left(\mathbb{R}^{3}, S, \mathbb{R}^{2}\right)$ is a comparison constellation with lower tangency on $[0, \infty)$.

Moreover, for $p \geq 2$,

$$
\mathcal{M}_{p}(r)=\frac{p}{r}+\frac{p-2}{r}=\frac{2(p-1)}{r} \geq 0,
$$

and it is straightforward to check that if we take $\rho>1$,

$$
\Lambda_{g, p}=\frac{\rho^{2} \ln (\rho)}{r \ln (r)}
$$

So,

$$
\int_{\rho}^{r} \Lambda_{g, p}(t) d t=\rho^{2} \ln (\rho) \ln \left(\frac{\ln (r)}{\ln (\rho)}\right)
$$

and consequently,

$$
\int_{\rho}^{\infty} \Lambda_{g, p}(r) d r=\infty
$$

Applying Theorem 4.1 we obtain that the Catenoid is $p$-parabolic for all $p \geq 2$.

Example 5.2. Let us consider now the Hyperboloid of one sheet. Now, the generating functions of this revolution surface are

$$
\begin{aligned}
& x(u)=\sqrt{1+u^{2}}, \\
& z(u)=u,
\end{aligned}
$$

and its parametrization is given by:

$$
\sigma(u, v)=\left(\sqrt{1+u^{2}} \cos v, \sqrt{1+u^{2}} \sin v, u\right) ; \quad u \in \mathbb{R} ; \quad v \in[-\pi, \pi] .
$$

Then, the distance function to $p=(0,0,0)$ is given by

$$
r(u, v)=\sqrt{1+2 u^{2}} .
$$

On the other hand, an easy computation shows that

$$
N(u, v)=\frac{1}{\sqrt{1+2 u^{2}}}\left(-\sqrt{1+u^{2}} \cos (v),-\sqrt{1+u^{2}} \sin (v), u\right),
$$


and then

$$
\begin{aligned}
\nabla^{S} r & =\nabla^{\mathbb{R}^{3}} r-\left\langle\nabla^{\mathbb{R}^{3}} r, N\right\rangle N \\
& =\frac{2 u}{\left(1+2 u^{2}\right)^{3 / 2}}\left(u \sqrt{1+u^{2}} \cos (v), u \sqrt{1+u^{2}} \sin (v), 1+u^{2}\right)
\end{aligned}
$$

So, the tangency function is given by

$$
\mathcal{T}(u, v)=\frac{2|u| \sqrt{1+u^{2}}}{1+2 u^{2}}
$$

Now, if we compute the second fundamental form of $S$ in $U_{r}(u, v)$ we obtain that

$$
\alpha\left(U_{r}, U_{r}\right)=-\frac{1}{\left(1+2 u^{2}\right)^{3 / 2}} N
$$

and then

$$
B(u, v)=-\frac{1}{\left(1+2 u^{2}\right)^{5 / 2}} .
$$

Finally, the mean curvature of the hyperboloid is given by

$$
H_{S}=-\frac{1}{2} \operatorname{div}^{S} N=\frac{u^{2}}{\left(1+2 u^{2}\right)^{3 / 2}},
$$

and consequently,

$$
\mathcal{C}(u, v)=\frac{u^{2}}{\left(2 u^{2}+1\right)^{5 / 2}}
$$

Since $u^{2}=\frac{r^{2}-1}{2}$ by equation (5.3), taking $\lambda(r)=-\frac{1}{r^{5}}, h(r)=\frac{r^{2}-1}{2 r^{5}}$ and $g(r)=\frac{\sqrt{r^{4}-1}}{r^{2}}$ we have that the triple $\left(\mathbb{R}^{3}, S, \mathbb{R}^{2}\right)$ is a comparison constellation with lower tangency on $[0, \infty)$.

Moreover, for $p \geq 2$,

$$
\mathcal{M}_{p}(r)=\frac{p\left(r^{4}+1\right)-\left(r^{2}+1\right)}{r^{5}} \geq 0
$$

because the parabola $f(x)=p x^{2}-x+(p-1)$ has its minimum in $x=\frac{1}{2 p}$, and $f\left(\frac{1}{2 p}\right) \geq 0$ when $p \geq 2$.

On the other hand, it is straightforward to check that if $\rho>1$, $\Lambda_{g, p}(r)=M(\rho)\left(\frac{r^{2}}{\sqrt{r^{2}-1}\left(1+r^{2}\right)^{\frac{p}{2(p-1)}}}\right) \geq M(\rho) \frac{r}{\left(1+r^{2}\right)^{\frac{p}{2(p-1)}}} \geq M(\rho) \frac{r}{1+r^{2}}$, where $M(\rho)$ is a positive constant and $p \geq 2$.

Therefore,

$$
\int_{\rho}^{\infty} \Lambda_{g, p}(t) d t \geq M(\rho) \int_{\rho}^{\infty} \frac{t}{1+t^{2}} d t=\infty
$$

and the hyperboloid of one sheet is $p$-parabolic for all $p \geq 2$ by Theorem 4.1 . 
Example 5.3. The Paraboloid is a revolution surface with generating curve given by:

$$
\begin{aligned}
& x(u)=u \\
& z(u)=\alpha u^{2}
\end{aligned}
$$

for $u \geq 0$ and any constant $\alpha \in \mathbb{R}$. The tangency function is

$$
\mathcal{T}(u, v)=\frac{1+2 \alpha^{2} u^{2}}{\sqrt{4 \alpha^{2} u^{2}+1} \sqrt{1+\alpha^{2} u^{2}}},
$$

and the corresponding distance function to the origin is

$$
r(u, v)=u \sqrt{1+\alpha^{2} u^{2}} .
$$

A straightforward computation give us that

$$
\begin{aligned}
B(u, v) & =\frac{2 \alpha^{2} u}{\sqrt{1+\alpha^{2} u^{2}}\left(1+4 \alpha^{2} u^{2}\right)^{2}} \geq 0, \\
\mathcal{C}(u, v) & =\frac{2 \alpha^{2} u\left(1+2 \alpha^{2} u^{2}\right)}{\sqrt{1+\alpha^{2} u^{2}}\left(1+4 \alpha^{2} u^{2}\right)^{2}} \geq 0 .
\end{aligned}
$$

Since $u^{2}=\left(\sqrt{1+\alpha^{2} r^{2}}-1\right) /\left(2 \alpha^{2}\right)$ by equation (5.4), the tangency function is a radial function. So, taking $\lambda(r)=0, h(r)=0$ and $g(r)=\mathcal{T}(u(r))$ we have that the triple $\left(\mathbb{R}^{3}, S, \mathbb{R}^{2}\right)$ is a comparison constellation with lower tangency on $[0, \infty)$.

Moreover, for $p \geq 2$,

$$
\mathcal{M}_{p}(r)=\frac{p}{r} \geq 0
$$

and it is easy to see that for $r$ bigger enough,

$$
g(r) \geq \sqrt{\frac{p \ln (r)}{1+p \ln (r)}} .
$$

As a consequence, for $\rho$ sufficiently large,

$$
\Lambda_{g, p} \geq\left(\frac{\rho^{p} \ln (\rho)}{r \ln (r)}\right)^{\frac{1}{p-1}}
$$

and

$$
\int_{\rho}^{\infty} \Lambda_{g, p}(t) d t \geq M(\rho) \int_{\rho}^{\infty} \frac{1}{(t \ln (t))^{\frac{1}{p-1}}} d t \geq M(\rho) \int_{\rho}^{\infty} \frac{1}{t \ln (t)} d t=\infty
$$

where $M(\rho)$ is a positive constant.

Then, $\int_{\rho}^{\infty} \Lambda_{g, p}(t) d t=\infty$ and applying Theorem 4.1, the Paraboloid is $p$-parabolic for all $p \geq 2$. 


\section{Proof of Theorem 4.1}

We define on model spaces $M_{w}^{m}$, the modified Laplacian

$$
\mathrm{L} \psi(x)=\Delta^{M_{w}^{m}} \psi(x)+\psi^{\prime}(r(x))\left(\frac{\mathcal{M}(r(x))}{(p-1) g^{2}(r(x))}-m \eta_{w}(r(x))\right),
$$

for smooth functions $\psi$ on $M_{w}^{m}$. If $\psi=\psi(r)$ only depends on the radial distance $r$, then

$$
\mathrm{L} \psi(r)=\psi^{\prime \prime}(r)+\psi^{\prime}(r)\left(\frac{\mathcal{M}(r)}{(p-1) g^{2}(r)}-\eta_{w}(r)\right) .
$$

Consider now the following Dirichlet-Poisson problem associated to L:

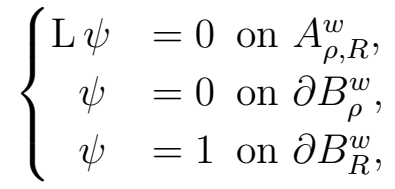

where $A_{\rho, R}^{w}$ is the annular domain in the model space defined as $A_{\rho, R}^{w}=$ $B_{R}^{w}-B_{\rho}^{w}$.

The explicit solution to the Dirichlet problem (6.2) is given in the following Proposition which is straightforward,

Proposition 6.1. The solution to the Dirichlet problem (6.2) only depends on $r$ and is given explicitly - via the function $\Lambda_{g, p}(r)$ introduced in Theorem 4.1, by:

$$
\psi_{\rho, R}(r)=\frac{\int_{\rho}^{r} \Lambda_{g, p}(t) d t}{\int_{\rho}^{R} \Lambda_{g, p}(t) d t} .
$$

The corresponding 'drifted' 2-capacity is

$$
\begin{aligned}
\operatorname{Cap}_{\mathrm{L}}\left(A_{\rho, R}^{w}\right) & =\int_{\partial D_{\rho}^{w}}\left\langle\nabla^{M} \psi_{\rho, R}, \nu\right\rangle d A \\
& =\operatorname{Vol}\left(\partial D_{\rho}^{w}\right) \Lambda_{g, p}(\rho)\left(\int_{\rho}^{R} \Lambda_{g, p}(t) d t\right)^{-1} .
\end{aligned}
$$

It is easy to see, using equation (6.3) and the balance condition (4.1) that

$$
\begin{aligned}
& \psi_{\rho, R}^{\prime}(r) \geq 0, \\
& \psi_{\rho, R}^{\prime \prime}(r)-\psi_{\rho, R}^{\prime}(r) \eta_{w}(r)=-\psi_{\rho, R}^{\prime}(r) \frac{\mathcal{M}(r)}{(p-1) g^{2}(r)} \leq 0 .
\end{aligned}
$$

Now, we need the following result, which relates the $p$-Laplacian of a radial function $f(r)$ with the operator L. 
Lemma 6.2. Let $\left\{N^{n}, S^{m}, M_{w}^{m}\right\}$ be a comparison constellation with lower tangency on $[0, R]$ for $R>0$. Let $f \circ r$ be a smooth real-valued function with $f^{\prime} \geq 0$, and suppose now that $f(r)$ satisfies the following condition:

$$
f^{\prime \prime}(r)-f^{\prime}(r) \eta_{w}(r) \leq 0 .
$$

Then, for all $x \in S$, and $p \geq 2$,

$$
\Delta_{p}^{S} f(r(x)) \leq(p-1) F^{p-2}(x) g^{2}(r(x)) \mathrm{L}(f(r(x))),
$$

where $L$ is the second order differential operator defined by equation (6.1) and $F$ is given by equation

$$
F(x)=f^{\prime}(r(x))\left\|\nabla^{S} r(x)\right\| .
$$

Proof. Computing as in $[\mathrm{HMP}]$, we have

$$
\begin{aligned}
& \Delta_{p}^{S} f(r(x))=F^{p-2}(x)\left(( p - 2 ) \left(f^{\prime \prime}(r(x))\left\|\nabla^{S} r(x)\right\|^{2}\right.\right. \\
& \left.\left.+f^{\prime}(r(x)) \frac{\left\langle\nabla^{S} r(x), \nabla^{S}\left\|\nabla^{S} r(x)\right\|\right\rangle}{\left\|\nabla^{S} r(x)\right\|}\right)+\Delta^{S} f(r(x))\right) .
\end{aligned}
$$

This partial 'isolation' of the factor $(p-2)$ is the reason behind the general assumption $p \geq 2$ in this work. Once we have equation (6.8), we argue as follows:

First, it is easy to see that

$$
\begin{aligned}
& \frac{\left\langle\nabla^{S} r(x), \nabla^{S}\left\|\nabla^{S} r(x)\right\|\right\rangle}{\left\|\nabla^{S} r(x)\right\|} \\
& \quad=\operatorname{Hess}^{N}(r(x))\left(U_{r}, U_{r}\right)+\left\langle\nabla^{N} r(x), \alpha_{x}\left(U_{r}, U_{r}\right)\right\rangle .
\end{aligned}
$$

This quantity is bounded from above using Theorem 3.14 and the lower bound of $\mathcal{B}(x)$. On the other hand, since the o-radial mean convexity of $S$, $\mathcal{C}(x)$, is bounded from below by the function $h(r(x))$, we obtain the following estimate using Proposition 3.17, (recall that $f^{\prime}(r) \geq 0$ )

$$
\Delta^{S}(f \circ r) \leq\left(f^{\prime \prime}(r)-f^{\prime}(r) \eta_{w}(r)\right)\left\|\nabla^{S} r\right\|^{2}+m f^{\prime}(r)\left(\eta_{w}(r)-h(r)\right) .
$$

So, using the fact that $f(r)$ satisfies inequality (6.6) and that $\left\|\nabla^{S}(r)\right\| \geq$ $g(r)$, we have

$$
\Delta_{p}^{S}(f(r(x))) \leq(p-1) F^{p-2}(x) g^{2}(r) \mathrm{L}(f(r)),
$$

as claimed in the lemma.

Now we transplant the model space solutions $\psi_{\rho, R}(r)$ of equation (6.2) into the extrinsic annulus $A_{\rho, R}=D_{R}(o) \backslash \bar{D}_{\rho}(o)$ in $S$ by defining

$$
\Psi_{\rho, R}: A_{\rho, R} \rightarrow \mathbb{R}, \quad \Psi_{\rho, R}(x)=\psi_{\rho, R}(r(x)) .
$$

Here $D_{\rho}(o)$ is an open precompact set, with smooth boundary $\partial D_{\rho}(o)$, (see Remark 3.2), and $D_{R}(o)$ is that component of $B_{R}(o) \cap S$ which contains 
$D_{\rho}(o)$. Then, we extend $\Psi_{\rho, R}$ to $\bar{D}_{\rho}(o)=S \cap \bar{B}_{\rho}(o)$ by setting $\Psi_{\rho, R}(x)=0$ for $x \in S \cap \bar{B}_{\rho}(o)$.

Since $\psi_{\rho, R}^{\prime}(r) \geq 0$ and $\mathrm{L} \psi_{\rho, R}=0$ in $A_{\rho, R}^{w}$, we obtain, applying Lemma 6.2 to the function $\Psi_{\rho, R}$,

$$
\Delta_{p}^{S} \Psi_{\rho, R} \leq 0 \quad \text { in } \quad D_{R}(o) \backslash \bar{D}_{\rho}(o),
$$

that is to say, $\Psi_{\rho, R}$ is a $p$-supersolution in $D_{R}(o) \backslash \bar{D}_{\rho}(o)$.

As $S$ is properly immersed, $D_{\rho}(o)$ and $D_{R}(o)$ are precompact and with regular boundary, so there exists a unique function $u \in C\left(\bar{D}_{R}(o)\right)$ which is $p$-harmonic in $D_{R}(o) \backslash \bar{D}_{\rho}(o)$ such that $u=0$ in $\bar{D}_{\rho}(o), u=1$ in $\partial D_{R}(o)$, and that

$$
\operatorname{Cap}_{p}\left(D_{\rho}(o), D_{R}(o)\right):=\operatorname{Cap}_{p}\left(\bar{D}_{\rho}(o), D_{R}(o)\right)=\int_{D_{R}(o)}\left\|\nabla^{S} u\right\|^{p} d \mu .
$$

(see [T1], [HKM, pp. 106-107], and [Gri, pp. 152]).

Furthermore, let $\Psi_{\rho, R}$ be the transplanted $p$-supersolution in $D_{R}(o)$. By the comparison principle, we have now

$$
u(x) \leq \Psi_{\rho, R}(x)
$$

for all $x \in D_{R}(o)$. Hence, as $u(x)=\Psi_{\rho, R}(x)=0$ for all $x \in \bar{D}_{\rho}(o)$, we obtain that

$$
\left\|\nabla^{S} u(x)\right\| \leq\left\|\nabla^{S} \Psi_{\rho, R}(x)\right\|
$$

for all $x \in \partial D_{\rho}(o)$.

With same arguments than in $[\mathrm{HMP}]$, but inverting all inequalities, we obtain

$$
\operatorname{Cap}_{p}\left(\bar{D}_{\rho}(o), D_{R}(o)\right) \leq\left(\frac{\operatorname{Cap}_{\mathrm{L}}\left(A_{\rho, R}^{w}\right)}{\operatorname{Vol}\left(\partial D_{\rho}^{w}\right)}\right)^{p-1} \int_{\partial D_{\rho}}\left\|\nabla^{S} r\right\|^{p-1} d \mathcal{H}^{m-1}
$$

As, on the other hand, $D_{\rho}(o)$ is precompact with a smooth boundary thence,

$$
0<\int_{\partial D_{\rho}}\left\|\nabla^{S} r\right\|^{p-1} d \mathcal{H}^{m-1}<\infty .
$$

So finally we have

$$
\begin{aligned}
& \operatorname{Cap}_{p}\left(\bar{D}_{\rho}(o), S^{m}\right)=\lim _{R \rightarrow \infty} \operatorname{Cap}_{p}\left(\bar{D}_{\rho}(o), D_{R}(o)\right) \leq \\
& \quad\left(\int_{\partial D_{\rho}}\left\|\nabla^{S} r\right\|^{p-1} d \mathcal{H}^{m-1}\right)\left(\lim _{R \rightarrow \infty} \frac{\operatorname{Cap}_{\mathrm{L}}\left(A_{\rho, R}^{w}\right)}{\operatorname{Vol}\left(\partial D_{\rho}^{w}\right)}\right)^{p-1}=0,
\end{aligned}
$$

since $\lim _{R \rightarrow \infty} \operatorname{Cap}_{\mathrm{L}}\left(A_{\rho, R}^{w}\right)=0$ by hypothesis (4.2) and equality (6.4). Thus $D_{\rho}(o)$ is an open precompact subset with zero $p$-capacity in $S^{m}$, and $p$ parabolicity of that submanifold follows. 


\section{Proof of Theorem 4.2}

We define the following modified Laplacian $\mathrm{L}$ on model spaces $M_{w}^{m}$,

$$
\mathrm{E} \phi(x)=\Delta^{M_{w}^{m}} \phi(x)+\phi^{\prime}(r(x))\left(\frac{\mathcal{M}(r(x))}{(p-1)}-m \eta_{w}(r(x))\right),
$$

for smooth functions $\phi$ on $M_{w}^{m}$. As before, if $\phi=\phi(r)$ only depends on the radial distance $r$, then

$$
\mathrm{€} \phi(r)=\phi^{\prime \prime}(r)+\phi^{\prime}(r)\left(\frac{\mathcal{M}(r)}{(p-1)}-\eta_{w}(r)\right) .
$$

Consider now the smooth radial solution $\phi_{\rho, R}(r)$ of the Dirichlet-Poisson problem associated to $\mathrm{E}$ and defined on the annulus $A_{\rho, R}^{w}=B_{R}^{w}-B_{\rho}^{w}$.

Now we transplant the model space solutions $\phi_{\rho, R}(r)$ of this problem into the extrinsic annulus $A_{\rho, R}=D_{R}(o) \backslash \bar{D}_{\rho}(o)$ in $S$ as in the proof of Theorem 4.1, so we have

$$
\Phi_{\rho, R}: A_{\rho, R} \rightarrow \mathbb{R}, \quad \Phi_{\rho, R}(x)=\phi_{\rho, R}(r(x)) .
$$

Using the lower bounds of $\mathcal{B}(x)$, and $\mathcal{C}(x)$, the fact that $\Phi_{\rho, R}^{\prime}(r) \geq 0$ and applying Theorem 3.14, and Proposition 3.17 we obtain, as we did for any radial function $f(r)$ satisfying $f^{\prime}(r) \geq 0$ in the proof of Lemma 6.2:

$$
\begin{aligned}
\Delta^{S} \Phi_{\rho, R} & \leq\left(\Phi_{\rho, R}^{\prime \prime}(r)-\Phi_{\rho, R}^{\prime}(r) \eta_{w}(r)\right)\left\|\nabla^{S} r\right\|^{2} \\
& +m \Phi_{\rho, R}^{\prime}(r)\left(\eta_{w}(r)-h(r)\right) .
\end{aligned}
$$

Hence, as

$$
\Phi_{\rho, R}^{\prime \prime}(r)-\Phi_{\rho, R}^{\prime}(r) \eta_{w}(r) \geq 0, \forall r>0
$$

because the balance condition (4.3) and $\left\|\nabla^{S} r\right\| \leq 1$, we obtain

$$
\Delta_{p}^{S}\left(\Phi_{\rho, R}(r(x))\right) \leq(p-1) F^{p-2}(x) g^{2}(r) \mathrm{L}\left(\Phi_{\rho, R}(r)\right)=0,
$$

where $F=\Phi_{\rho, R}^{\prime}(r(x))\left\|\nabla^{S} r(x)\right\|$. The rest of the proof follows in the same way than in Section 6.

\section{REFERENCES}

[E] Evans, L.C., A new proof of local $C^{1, \alpha}$ regularity for solutions of certain degenerate elliptic p.d.e., J. Differential Equations, 45, 3, (1982), 356-373.

[EP] A. Esteve, V. Palmer On the characterization of parabolicity in Real Space Forms, Preprint 2009

[GT] V. Gol'dshtein and M. Troyanov, The Kelvin-Nevanlinna-Royden criterion for p-parabolicity, Math. Z. 232, (1999), 607-619.

[GreW] R. Greene and $\mathrm{H}$. Wu, Function theory on manifolds which possess a pole, Lecture Notes in Math., vol. 699, Springer-Verlag, Berlin and New York (1979).

[Gri] A. Grigor'yan, Analytic and geometric background of recurrence and nonexplosion of the Brownian motion on Riemannian manifolds, Bull. Amer. Math. Soc. 36 (1999), 135-249. 
[HKM] J. Heinonen, T. Kilpeläinen, and O. Martio, Nonlinear potential theory of degenerate elliptic equations, The Clarendon Press, Oxford University Press, New York, 1993. Oxford Science Publications.

[HMP] I. Holopainen, S. Markvorsen and V. Palmer, p-capacity and p-hyperbolicity of submanifolds, Revista Matemática Iberoamericana, To appear.

[Li] Lieberman, G. M., Boundary regularity for solutions of degenerate elliptic equations, Nonlinear Anal. 12, 11, (1988), 1203-1219.

[LS] T. Lyons and D. Sullivan, Function theory, random paths and covering spaces, J. Diff. Geometry 19 (1984), 299-323.

[MP1] S. Markvorsen and V. Palmer, Extrinsic isoperimetric analysis on submanifolds with curvatures biunded below, Journal of Geometric Analysis, To appear.

[Se] J. Serrin, Local behavior of solutions of quasi-linear equations, Acta Math. 111 (1964), 247-302.

[T1] M. Troyanov, Parabolicity of Manifolds, Siberian Advances in Mathematics 9 (1999) 125-150.

Departamento de Geometría y Topología, Universidad de Granada, Granada, SPAIN.

E-mail address: ahurtado@ugr.es

Departament de Matemàtiques, Universitat Jaume I, Castelló, Spain.

E-mail address: palmer@mat.uji.es 